\title{
Conjunctival epithelial hyperplasia in a patient with a nodular lesion in the palpebral conjunctiva: A case report
}

\author{
SHIGERU KAWABATA ${ }^{1}$, YAMATO YOSHIKAWA ${ }^{2}$, YOSHITAKA KURISU ${ }^{1}$, \\ KENSUKE TAJIRI $^{2}$, TERUYO KIDA ${ }^{2}$ and YOSHINOBU HIROSE ${ }^{1}$
}

\begin{abstract}
Departments of ${ }^{1}$ Pathology and ${ }^{2}$ Ophthalmology, Faculty of Medicine, Osaka Medical and Pharmaceutical University, Takatsuki, Osaka 569-8686, Japan
\end{abstract}

Received November 26, 2021; Accepted January 18, 2022

DOI: $10.3892 /$ br.2022.1507

\begin{abstract}
The conjunctiva is a thin and delicate mucous membrane lining the inner eyelid and the anterior surface of the eyeball. Although hyperplastic changes can occur due to nonspecific chronic inflammation, 'conjunctival epithelial hyperplasia' has not been sufficiently established as a pathological entity. Additionally, the immunohistochemical (IHC) features of both the intact conjunctiva epithelium and conjunctival epithelial hyperplasia have not been sufficiently evaluated. The present report describes the case of an 86-year-old man who consulted with an ophthalmologist for a 6-month-old nodular lesion on his left eye. Located in the medial aspect of the left lower palpebral conjunctiva, the lesion was slightly erythematous and smooth. An excisional biopsy of the lesion was performed to obtain a pathological diagnosis. The hematoxylin and eosin sections revealed a thickened conjunctival epithelium composed of hyperplastic cuboidal epithelial cells and goblet cells, indicating conjunctival epithelial hyperplasia. Atypia, increased mitosis and a papillomatous architecture, indicative of neoplastic changes, were not observed. This resulted in conjunctival squamous intraepithelial neoplasia and squamous cell papilloma being ruled out. IHC analysis was performed to further characterize the lesion as well as the intact conjunctival epithelium. The thick conjunctival epithelium was composed of epithelial cells that stained positive for cytokeratin [AE1/AE3 (intensity: +), CK5/6 (intensity: ++), and CK7 (intensity: +)] and p63-positive basal cells (intensity: + ) whose presence in the conjunctiva has received insufficient recognition. Moreover, squamous metaplasia was found in a segment of the thick conjunctiva, which exhibited IHC features similar to those of hyperplasia. CK5/6 was positive, indicating endogenous squamous differentiation
\end{abstract}

Correspondence to: Dr Shigeru Kawabata, Department of Pathology, Faculty of Medicine, Osaka Medical and Pharmaceutical University, 2-7 Daigakumachi, Takatsuki, Osaka 569-8686, Japan E-mail: shigeru.kawabata@ompu.ac.jp

Key words: ophthalmic pathology, conjunctival epithelium, hyperplasia of the conjunctival epithelial hyperplasia. These findings led to the diagnosis of conjunctival epithelial hyperplasia as a pathological entity. Further collection and analysis of several cases of conjunctival epithelial hyperplasia may lead the development of preventative methods and drug treatments for this lesion, and additional prognostic data, such as the recurrence rate.

\section{Introduction}

The conjunctiva is a thin and delicate mucous membrane, lining the inner eyelid and the anterior surface of the eyeball. Histologically, the conjunctival epithelium is composed of two to five layers of columnar or cuboidal cells, mucin-secreting goblet cells and melanocytes (1). Since the ocular surface is exposed to numerous infectious and noninfectious agents or allergens, hyperplastic changes can occur due to nonspecific chronic inflammation of the conjunctiva (2). Ohashi et al (3) reported of two cases of conjunctival mucoepithelial hyperplasia amongst elderly patients. Specifically, nodular lesions found on the internal canthus were presented in both patients. Clinically, neoplasms were identified as differential diagnoses in both cases; however the nodular lesions were pathologically characterized by goblet cell hyperplasia and chronic inflammation of the stroma. For neoplasms as differential diagnoses, conjunctival squamous intraepithelial neoplasia (CSIN) and squamous cell papilloma of the conjunctiva were more probable diseases. CSIN shows dysplastic changes of the conjunctiva, located at the limbus within the interpalpebral area, and is the most common form of ocular surface neoplasia (4). Squamous cell papilloma of the conjunctiva is a benign neoplasm characterized by an outgrowth of epithelial and stromal elements of the conjunctiva; it is often associated with human papilloma virus (HPV), and is more frequently found in men and amongst patients aged 20-29 years (5). Although there are some case reports of hyperplastic lesions in the conjunctiva, as a pathological entity, conjunctival epithelial hyperplasia has not been sufficiently established. Additionally, the immunohistochemical (IHC) features of both the intact conjunctiva epithelium and conjunctival epithelial hyperplasia have not been sufficiently evaluated. In the present report, the case of a nodular lesion in the palpebral conjunctiva is described. The component cells in the conjunctival epithelium exhibited 
hyperplastic changes with minimal inflammation, and showed squamous metaplasia in a segment of thick conjunctiva. IHC analysis was performed to further characterize the immunophenotype of the lesion and rule out differential diagnoses, such as CSIN and squamous cell papilloma of the conjunctiva.

\section{Case report}

An 86-year-old man consulted with an ophthalmologist for a 6-month-old nodular lesion on his left eye. Although the nodular lesion had not grown during these 6 months, he started feeling uncomfortable due to an increase in discharge from the eye and blurring of vision. There were no other changes, such as failing vision or limited eye movement. His past medical history and family history were unremarkable. He had no allergies, did not smoke and did not drink alcohol. The visual acuities [normal $>1.0$ (6)] of the right and left eye were 0.8 and 1.2 , respectively, indicating a mild decline in the acuity of the right eye. Both intraocular pressures were $14 \mathrm{mmHg}$, which falls within the normal range [10-21 mmHg (7)]. Laboratory tests showed no abnormalities, except for C-reactive protein (CRP) level, which was $0.15 \mathrm{mg} / \mathrm{dl}[0.00-0.14 \mathrm{mg} / \mathrm{dl}(8)]$. Located in the medial aspect of the left lower palpebral conjunctiva, the lesion was slightly erythematous and smooth (Fig. 1, black arrowhead). Although CRP level was slightly elevated, based on the nodularity of the lesion, hyperplasia rather than inflammation was a more probable differential diagnosis. An excisional biopsy of the lesion was performed to obtain a pathological diagnosis.

The tissue specimen was fixed in $10 \%$ neutral buffered formalin, and then embedded in paraffin wax for hematoxylin and eosin (H\&E) staining. IHC analysis was performed using Vectastain Elite ABC kits from Vector Laboratories, Inc., according to the manufacturer's instructions for blocking, secondary antibody dilution and labeling. The following primary antibodies against cytokeratin (CK) markers were purchased: AE1/AE3 (clone AE1/AE3; Nichirei); CK5/6 (clone D5/16 B4), CK7 (clone OV-TL 12/30), and CK20 (Clone Ks20.8) all from Dako (Agilent Technologies, Inc.); and anti p63 (clone 4A4; Nichirei) antibody, a myoepithelial marker. The anti Ki-67 (clone MIB-1) antibody was used to evaluate cell proliferation using a Dako system (Agilent Technologies, Inc.). DAB (Sigma-Aldrich; Merck KGaA) was freshly prepared from tablets for chromogenic staining at $20^{\circ} \mathrm{C}$ for $10 \mathrm{~min}$.

The H\&E stained section of the intact part of the palpebral conjunctiva showed a stratified epithelium composed of two to seven layers of cuboidal cells with mucin-producing goblet cells (Fig. 2). AE1/AE3 (intensity: +), CK5/6 (intensity: +) and CK7 (intensity: +) stained positively in the epithelium (Fig. 2), whereas CK20 (intensity: -) was negative (data not shown). p63-positive cells were detected in the basal and parabasal layers (intensity: + ). This indicated that the conjunctival epithelium had basal cells similar to that of the respiratory epithelium. Additionally, the proliferative zone of the epithelium was located in the basal and parabasal layers, as indicated by Ki-67-positive staining (Fig. 2).

The H\&E staining of the nodule revealed a thickened conjunctival epithelium, composed of increased cuboidal epithelial cells and goblet cells (Fig. 3A). Atypia and increased mitosis, indicative of neoplastic changes, were not observed on

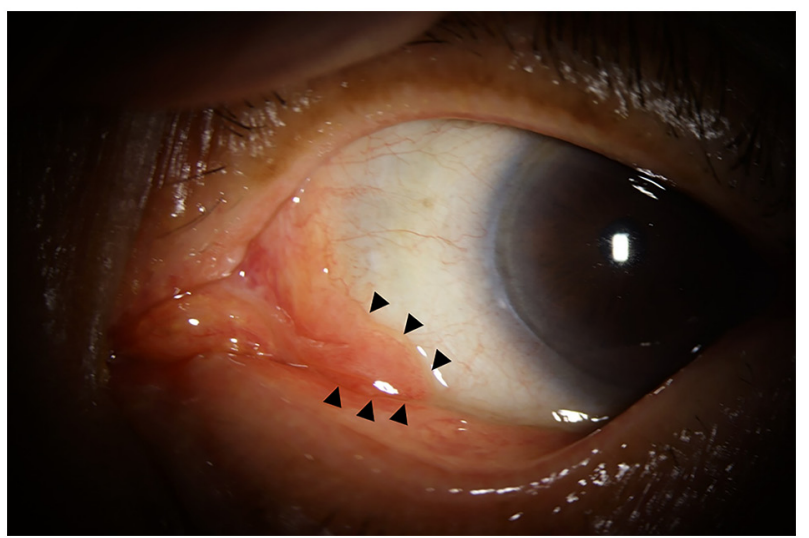

Figure 1. A nodular lesion in the medial aspect of the left lower palpebral conjunctiva (black arrowhead).

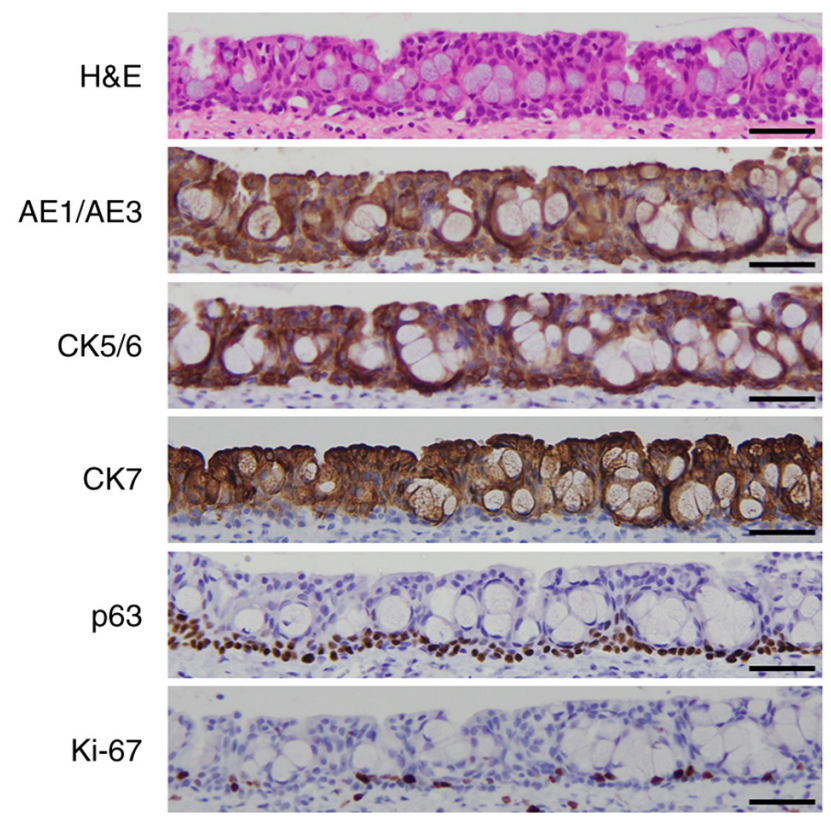

Figure 2. Intact conjunctival epithelium. The stratified columnar epithelium was composed of two to seven layers of cuboidal cells with mucin-producing goblet cells in hematoxylin and eosin (H\&E) section. The indicated markers were assessed by immunohistochemical analyses. Magnification, x200. Scale bar, $50 \mu \mathrm{m}$

high-magnification microscopy (Fig. 3B). As shown in Fig. 4, the thick conjunctival epithelium consisted of epithelial cells that stained positive for CK [AE1/AE3 (intensity: +), CK5/6 (intensity: ++), and CK7 (intensity: +)] and p63-positive basal cells (intensity: + ). There were no large sections of Ki-67-positive cells in the epithelium, so a tumorous lesion was unlikely. These findings suggested hyperplasia of the thick conjunctival epithelium. In Fig. 5, squamous metaplasia was detected in a segment of the thick conjunctiva, the IHC features of which were similar to those of hyperplasia (Fig. 4).

\section{Discussion}

Hyperplastic changes were detected in the cells comprising the nodular lesion of the conjunctival epithelium. IHC analysis was also performed. Ohashi et al (3) reported conjunctival 

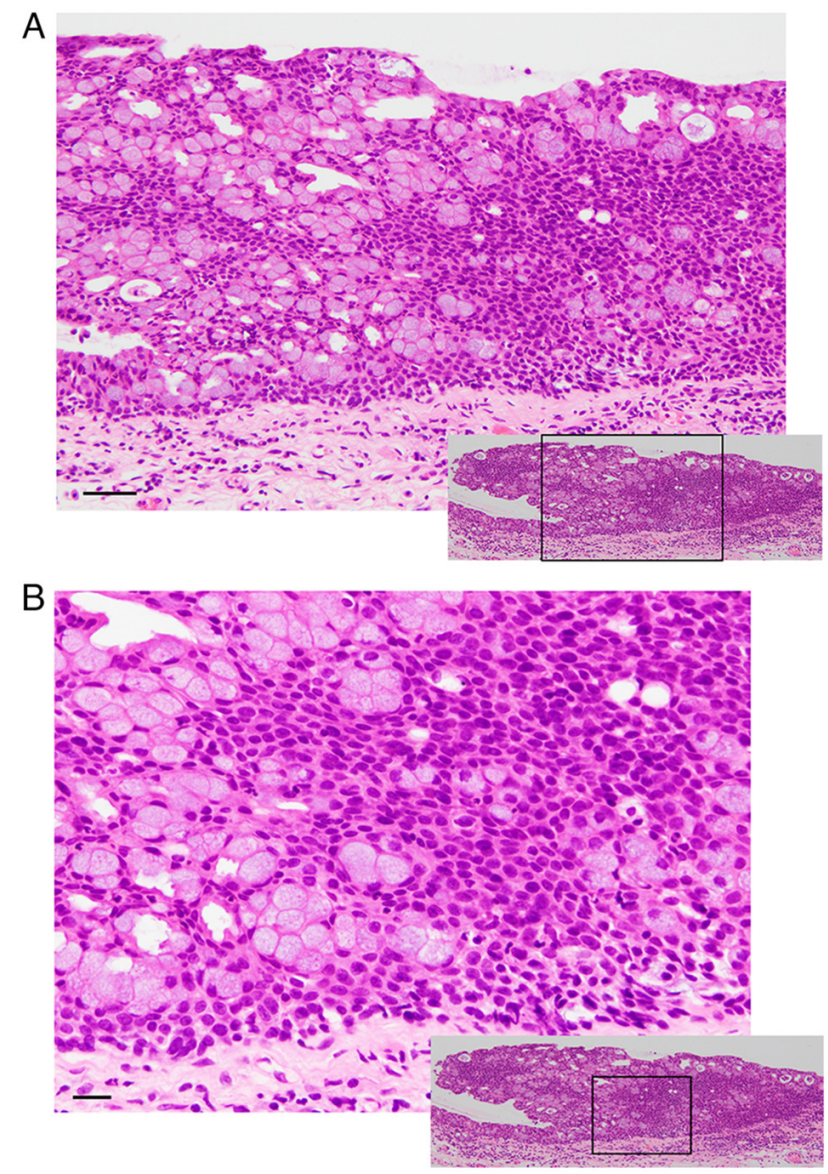

Figure 3. Conjunctival epithelial hyperplasia. Hematoxylin and eosin section revealed thickened conjunctival epithelium, composed of increased cuboidal epithelial cells and goblet cells: (A) x200 magnification; scale bar, $50 \mu \mathrm{m}$. (B) $\times 400$ magnification; scale bar, $20 \mu \mathrm{m}$. The larger figure shows a magnified version of the black lined insets.

mucoepithelial hyperplasia amongst the elderly. These cases were characterized by goblet cell hyperplasia with nonspecific chronic inflammation that mimicked neoplastic lesions. In the present case, hyperplasia was observed not only in the goblet cells but also in epithelial and basal cells of the nodule. These findings led to the diagnosis of conjunctival epithelial hyperplasia as a pathological entity, which is a diagnosis that has not been sufficiently established yet.

As they are amongst the differential diagnoses for the described lesion, CSIN and squamous cell papilloma of the conjunctiva had to be ruled out. CSIN represents the in situ precursor lesion of squamous cell carcinoma, which shows acanthosis with loss of goblet cells, atypical keratinocytes and suprabasilar mitotic figures. Immunophenotype of CSIN is AE1/AE3 positive, but shows low expression of CK7. Usually, expression of p53 and $\mathrm{Ki}-67$ is increased and appear in suprabasilar cells (4). In this described lesion, loss of goblet cells, atypia, increased mitosis, decreased CK7 expression and a broad distribution of $\mathrm{Ki}$-67-positive cells were absent. Additionally, expression of p53 was not increased, indicating wild-type pattern (data not shown). Based on this data, CSIN was excluded. Squamous cell papilloma of the conjunctiva is a benign lesion caused by HPV and is typically an exophytic growth with papillary proliferation of stratified squamous epithelium. Koilocytosis (nuclear pyknosis and cytoplasmic

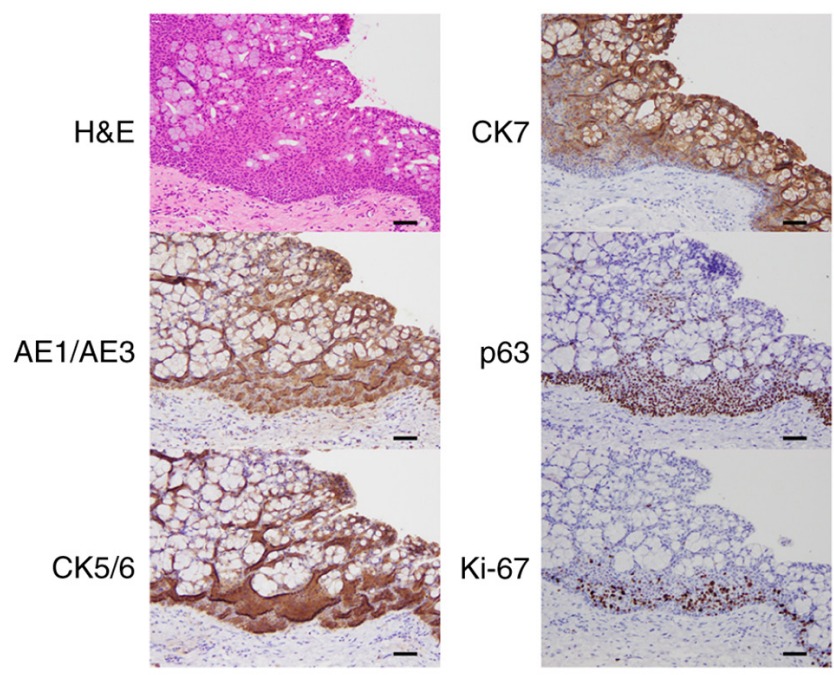

Figure 4. Characterization of conjunctival epithelial hyperplasia. The indicated markers were assessed by immunohistochemical analyses. Magnification, x200. Scale bar, $50 \mu \mathrm{m}$. H\&E, hematoxylin and eosin.

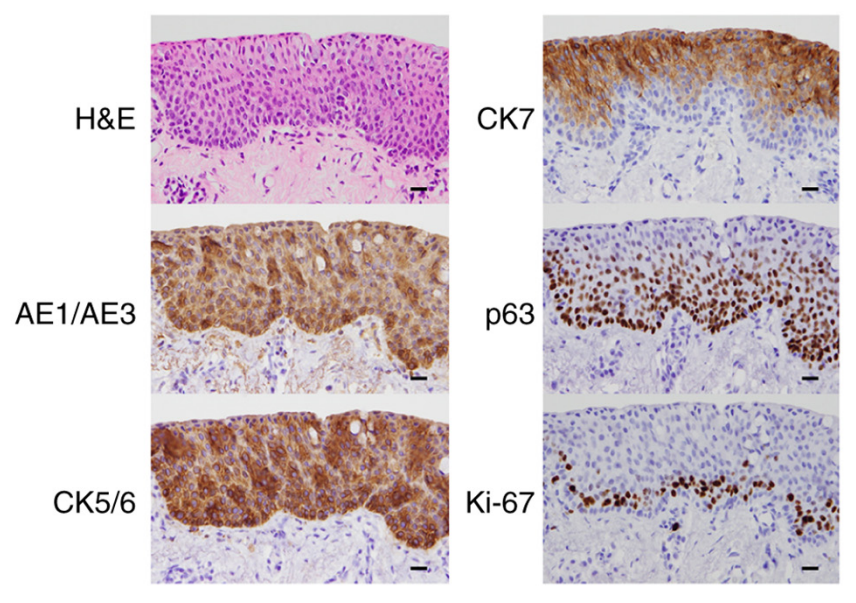

Figure 5. Characterization of the squamous metaplasia noted in a segment of the conjunctival epithelial hyperplasia. The indicated markers were assessed by immunohistochemical analyses. Magnification, $\mathrm{x} 400$. Scale bar, $20 \mu \mathrm{m}$. H\&E, hematoxylin and eosin.

clearing) is a morphological hallmark of HPV infection (5). This lesion showed neither papillomatous architecture nor koilocytosis, and squamous cell papilloma was ruled out.

On immunohistochemistry, the epithelial cells in both the intact conjunctiva and conjunctival epithelial hyperplasia positively stained for AE1/AE3, CK5/6 and CK7, but not CK20. AE1/AE3 antibody is a CK cocktail, referred to as a 'pancytokeratin'. It detects both high (CK1-6, 10, 14, 15 and 16) and low (CK7, 8, and 19) molecular weight keratins (9). CK5/6 (high molecular weight keratins) antibody detects stratified and transitional epithelium, proliferating squamous epithelium and mesothelial cells. The CK7 (low molecular weight keratin) antibody detects non-keratinizing epithelia, except for those of the intestine, ectocervix, prostate, and liver. CK20 is a low-molecular-weight keratin that identifies the gastrointestinal and urothelial epithelia (10-12). The IHC features of the intact conjunctiva and conjunctival epithelial hyperplasia are similar to those of respiratory epithelium in that both are 
CK7-positive and CK20-negative. CK5/6 was also positive in this case, indicating endogenous squamous differentiation of the intact conjunctiva and conjunctival epithelial hyperplasia.

Based on previous literature, the presence of basal cells in the conjunctiva has received insufficient recognition. The presence of p63, which is expressed in the basal epithelia of multiple organs (13), was evaluated in the intact conjunctiva. As shown in Fig. 2, the basal and parabasal layers positively stained for p63. Ramalho et al (14) assessed the relationship between p63 and p16 expression in primary and recurrent pterygia and showed p63 positivity in the basal layer of the normal conjunctiva. The results of the present case support the findings of Ramalho et al (14), indicating the presence of basal cells as well as respiratory epithelium (15).

In conclusion, the results of this investigation suggest that 'conjunctival epithelial hyperplasia' should be considered a pathological entity and indicate the presence of basal cells in the conjunctiva. Further collection and analysis of other cases of conjunctival epithelial hyperplasia can help elucidate its characteristics, including prognostic data such as the recurrence rate, and help in the development of prevention methods and therapeutic drugs for this lesion.

\section{Acknowledgements}

The authors would like to thank Research Scientist Yusuke Onishi (Department of Pathology, Faculty of Medicine, Osaka Medical and Pharmaceutical University, Osaka, Japan) for technical support with the IHC analyses.

\section{Funding}

No funding was received.

\section{Availability of data and materials}

The datasets used and/or analyzed during the present study are available from the corresponding author on reasonable request.

\section{Authors' contributions}

SK designed this study, collected and analyzed data, and wrote the manuscript; YY, KT and TK contributed to clinical data acquisition and interpretation; $\mathrm{YK}$ and $\mathrm{YH}$ evaluated the pathological findings and approved the final pathological diagnosis. All authors have read approved the final manuscript. SK and YK confirm the authenticity of all the raw data.

\section{Ethics approval and consent to participate}

Our institute does not require an approval for a case report based on Ethical Guidelines for Medical and Health Research Involving Human Subjects from the Japanese Ministry of Health, Labour and Welfare. The patient provided written informed consent.

\section{Patient consent for publication}

Not applicable.

\section{Competing interests}

The authors declare that they have no competing interests.

\section{References}

1. Proia AD and Cummings TJ: In: Mills SE (ed). Histology for pathologists, 5th edition. Philadelphia, Wolters Kluwer, pp335-361, 2020.

2. Eagle RC Jr: Conjunctiva. In: Eye Pathology: An Atlas and Text, 3rd edition. Philadelphia, Wolters Kluwer, pp90-133, 2016. Available from: https://www.proquest.com/legacydocview/EBC/5228949?accountid=38219.

3. Ohashi A, Elsayed AA, Yasuma T, Iijima T and Nakamura S: Conjunctival mucoepithelial hyperplasia of the elderly. Int Ophthalmol 35: 611-614, 2015.

4. Reese A and Margo CE: Conjunctival squamous intraepithelial neoplasia and its differential diagnosis. J Clin Pathol, Feb 22 , 2021 (Epub ahead of print).

5. Kalogeropoulos C, Koumpoulis I, Papadiotis E, Zioga A, Gkrepi K, Pappa C, Paschides C, Malamou-Mitsi V and Aspiotis M: Squamous cell papilloma of the conjunctiva due to human papillomavirus (HPV): Presentation of two cases and review of literature. Clin Ophthalmol 6: 1553-1561, 2012.

6. Huang L, Kawasaki H, Yasuda R and Sakai R: Relationship between visual acuity and lifestyle: A cross-sectional study in Japanese children. Hiroshima J Med Sci 67: 105-111, 2018.

7. Wang YX, Xu L, Wei WB and Jonas JB: Intraocular pressure and its normal range adjusted for ocular and systemic parameters. The Beijing eye study 2011. PLoS One 13: e0196926, 2018.

8. Ozaka S, Kodera T, Ariki S, Kobayashi T and Murakami K: Acute pancreatitis soon after COVID-19 vaccination: A case report. Medicine (Baltimore) 101: e28471, 2022.

9. BadzioA,CzapiewskiP,Gorczyński A,Szczepańska-MichalskaK, Haybaeck J, Biernat W and Jassem J: Prognostic value of broad-spectrum keratin clones AE1/AE3 and CAM5.2 in small cell lung cancer patients undergoing pulmonary resection. Acta Biochim Pol 66: 111-114, 2019.

10. Moll R, Franke WW, Schiller DL, Geiger B and Krepler R: The catalog of human cytokeratins: Patterns of expression in normal epithelia, tumors and cultured cells. Cell 31: 11-24, 1982.

11. Bragulla HH and Homberger DG: Structure and functions of keratin proteins in simple, stratified, keratinized and cornified epithelia. J Anat 214: 516-559, 2009.

12. Karantza V: Keratins in health and cancer: More than mere epithelial cell markers. Oncogene 30: 127-138, 2011.

13. Dewar R, Fadare O, Gilmore H and Gown AM: Best practices in diagnostic immunohistochemistry: Myoepithelial markers in breast pathology. Arch Pathol Lab Med 135: 422-429, 2011.

14. Ramalho FS, Maestri C, Ramalho LNZ, Ribeiro-Silva A and Romão E: Expression of p63 and p16 in primary and recurrent pterygia. Graefe's Arch Clin Exp Ophthalmol 244: 1310-1314, 2006.

15. Steurer S, Riemann C, Büscheck F, Luebke AM, Kluth M, Hube-Magg C, Hinsch A, Höflmayer D, Weidemann S, Fraune C, et al: $\mathrm{p} 63$ expression in human tumors and normal tissues: A tissue microarray study on 10,200 tumors. Biomark Res 9: 7, 2021. 